\title{
Measurement of strains in zircon inclusions by Raman spectroscopy
}

\author{
Claudia STANGARONE ${ }^{1, \#}$, Ross J. ANGEL ${ }^{1, *}$, Mauro PRENCIPE ${ }^{2}$, Nicola CAMPOMENOSI $^{3}$, \\ Boriana MIHAILOVA ${ }^{4}$ and Matteo ALVARO ${ }^{1}$
}

\author{
${ }^{1}$ Department of Earth and Environmental Sciences, University of Pavia, Via A. Ferrata 1, 27100 Pavia, Italy \\ *Corresponding author, e-mail: rossjohnangel@gmail.com \\ ${ }^{2}$ Department of Earth Sciences, University of Torino, Via Valperga Caluso 35, 10125 Torino, Italy \\ ${ }^{3}$ Department of Earth Science, Environment and Life, University of Genoa, Corso Europa 26, 16132 Genoa, Italy \\ ${ }^{4}$ Department of Earth Sciences, University of Hamburg, Grindelallee 48, 20146 Hamburg, Germany \\ \#Current address: Institute for Planetary Research, Deutsches Zentrum für Luft- und Raumfahrt (DLR), \\ Rutherfordstraße 2, 12489 Berlin, Germany
}

\begin{abstract}
We have carried out ab initio hybrid Hartree-Fock/Density Functional Theory simulations to determine the structure and vibrational modes of zircon, $\mathrm{ZrSiO}_{4}$, as a function of different applied strains. The changes in phonon-mode wavenumbers are approximately linear in the unit-cell strains, and have been fitted to determine the components of the phonon-mode Grüneisen tensors of zircon which reproduce the change in measured Raman shifts with pressure. They can therefore be used to convert Raman shifts measured from zircon inclusions in metamorphic rocks into strains that in turn can be used to determine the metamorphic conditions at the time that the inclusion was trapped. Due to the strong anisotropy in the thermal pressure of zircon, the phonon-mode Grüneisen tensor is not able to reproduce the temperature-induced changes in Raman shifts. Because zircon inclusions are normally measured at room conditions this does not prevent the calculation of their entrapment conditions.
\end{abstract}

Key-words: zircon; inclusions; elastic barometry; Raman spectroscopy; Grüneisen tensor.

\section{Introduction}

The discovery of coesite inclusions in garnet in the DoraMaira massif (Chopin, 1984) was "the little mineral that changed everything" (Tremblay, 2013). The discovery clearly indicated that continental crust can experience pressures in excess of $2.8 \mathrm{GPa}$ and be subsequently exhumed. The question of whether these rocks experienced lithostatic pressures of $2.8 \mathrm{GPa}$ and thus depths of metamorphism of $90 \mathrm{~km}$ or more, or whether instead they experienced significant deviatoric stress that stabilised coesite with respect to quartz at much shallower depths, was raised almost immediately by Smith (1984) in his description of coesite inclusions in pyroxenes from the Western Gneiss Region of Norway. The origin of such over-pressures could be on the microscale, generated by grain-grain interactions between minerals with different thermo-elastic properties, on the scale of the outcrop as indicated by the strongly heterogeneous deformation of the Dora-Maira rocks at peak conditions (Henry et al., 1993) or be regional in scale due to tectonic overpressure (e.g., Schmalholz \& Podladchikov, 2014; Gerya, 2015).

Conventional thermo-barometry is challenged in ultrahigh-pressure metamorphic (UHPM) terrains because it relies on thermodynamic equilibrium being attained in the rocks, and the textures and peak phase assemblage being preserved during exhumation. A complementary method of geobarometry, based on the stress state of inclusions in high-pressure minerals, has been developed over the past two decades, based on the original ideas of Rosenfeld \& Chase (1961). The basic idea is that a mineral such as coesite or zircon trapped inside a garnet that has grown at UHPM conditions will exhibit residual stresses when examined at room conditions, as a result of the contrast in thermoelastic properties (the equations of state [EoS]) of the host and the inclusion mineral. In simple terms, the inclusion mineral is constricted by the host and cannot expand freely on exhumation as would a crystal of the same mineral in the rock matrix. Knowledge of the EoS of the minerals allows possible entrapment conditions of the inclusion to be calculated and thus provides a second type of geobarometer completely independent of chemical equilibrium (Rosenfeld \& Chase, 1961). Recent developments in theory have expanded the original isotropic models for calculating entrapment conditions (e.g., Gillet et al., 1984; Zhang, 1998; Angel et al., 2014) to allow for the effects of the shapes and the local environment of the inclusions (Campomenosi et al., 2018; Mazzucchelli et al., 2018). The effects of the anisotropy of the inclusion minerals trapped within a host that is elastically isotropic can also be calculated by extension of these 
methods. Calculations of entrapment conditions are based on the strain state of the inclusion mineral still buried in its host relative to a free crystal of the same mineral at room conditions. The challenge is that small faceted inclusions can exhibit significant strain gradients (e.g., Campomenosi et al., 2018; Murri et al., 2018) and these cannot be measured by $\mathrm{X}$-ray laboratory diffraction because the large X-ray beams illuminate all or most of the inclusion and therefore return some average value of the unit-cell parameters of the inclusion and hence the strains. Such average values cannot be used to determine entrapment conditions of the inclusion. As shown in the original work on the coesite inclusions (Chopin, 1984; Smith, 1984), Raman spectra can be collected from much smaller volumes (of the order of $1 \mu \mathrm{m}^{3}$ ) and allow local strains to be determined if the values of the phonon-mode Grüneisen tensors for the inclusion phase are known (Angel et al., 2019). The values of the components of these tensors can be determined by calculating the Raman spectra of the inclusion mineral under different strain conditions with Density Functional Theory (DFT) (Murri et al., 2018, 2019). Coesite, being monoclinic, is a challenge for this approach because it requires many more simulations of different strain states to be performed than are required for a uniaxial mineral such as quartz, and the theory for calculating the elastic relaxation of monoclinic mineral inclusions has yet to be developed. However, zircon is also ubiquitous as an inclusion mineral in garnets in UHPM rocks such as those found in Dora-Maira (Schertl et al., 1991), and has the potential to be used as an elastic geobarometer as well as providing the opportunity for radiometric dating of the time of entrapment. In this paper we present the results of DFT simulations of zircon under a range of deviatoric strains in order to determine the components of the phonon-mode Grüneisen tensors of zircon. We show that these values correctly predict the change in the Raman shifts of modes under $P$, but not under $T$, and we show that this contrast in behaviour to that of quartz is due to differences between the anisotropy of the thermoelastic properties of the two minerals. The resulting tensor components can be used to determine the strains in zircon inclusions from measurements of their Raman spectra and thus ultimately their entrapment conditions.

\section{Computational details}

The geometry of the zircon structure was optimised by $a b$ initio HF/DFT simulations that were performed with the CRYSTAL17 code (Dovesi et al., 2018) using the same hybrid WC1LYP Hamiltonian (Wu \& Cohen, 2006), basis sets and parameters that were used for previous simulations of zircon under hydrostatic pressure (Stangarone et al., 2019). One full optimization of both the unit-cell parameters and the atomic positions was performed at zero pressure without any constraints except those imposed by the $I 4_{1}$ /amd space-group symmetry. This provided the unstrained reference structure. Atomic fractional coordinates of zircon were then optimized at 47 different strained configurations with fixed unit-cell parameters and the $a$ and $b$ unit-cell parameters kept equal to maintain the tetragonal symmetry. The unit-cell parameters were chosen to cover the range of strains, $\varepsilon_{1}=\Delta a / a, \varepsilon_{2}=\Delta b / b$, and $\varepsilon_{3}=\Delta c / c$, expected to be found in zircon inclusions in garnets. All of the computed geometries are at static equilibrium (i.e., at $0 \mathrm{~K}$, with no vibrational effects included). For each optimized geometry the normal modes of vibration and their wavenumbers were calculated within the limit of the harmonic approximation as described in Stangarone et al. (2019). The average mismatch between phonon-mode wavenumbers calculated in this way and experimental data for a wide variety of minerals is $5 \mathrm{~cm}^{-1}$ (Prencipe, 2019). The calculated phonon-mode frequencies and the structural data (atomic coordinates and unit-cell parameters) for the 48 optimisations of zircon are reported in the crystallographic information file $(C I F)$ deposited as Supplementary Material linked to this article and freely available at https://pubs.geoscienceworld.org/eurjmin/.

\section{Results}

\subsection{Structure}

The structure of zircon contains $\mathrm{Si}$ in tetrahedral coordination by oxygen, and $\mathrm{Zr}$ in 8 -fold coordination by oxygen in the form of triangular-faced dodecahedra (Fig. 1). The Si and $\mathrm{Zr}$ atoms occupy positions that are fixed by the $I 4_{1} /$ amd space-group symmetry. The $\mathrm{Si}$ and $\mathrm{Zr}$ are separated along [001] by a distance of exactly $c / 2$, and their coordination polyhedra are joined by a shared $\mathrm{O}-\mathrm{O}$ edge which form continuous columns of edge-sharing polyhedra which are parallel to the c-axis. The $\mathrm{Zr}-\mathrm{O}$ bonds to the $\mathrm{O}$ atoms in the shared edge are $0.13 \AA$ longer than those to O not involved in edgesharing with the $\mathrm{SiO}_{4}$, and the $\mathrm{O}-\mathrm{Si}-\mathrm{O}$ angle to the same shared edge is only $96.5^{\circ}$ (e.g., Kolesov et al., 2001; Finch \& Hanchar, 2003). These structural features indicate that there is a strong repulsive interaction between the neighbouring $\mathrm{Zr}$ and $\mathrm{Si}$ atoms along [001] (e.g., Kolesov et al., 2001), which makes this direction more than twice as stiff as the perpendicular directions under high pressure (e.g., Hazen \& Finger, 1979; Pina-Binvignat et al., 2018).

In the absence of experimental data for the structure and unit-cell parameters of zircon under deviatoric stress and strain, the only test of the validity of our DFT results is to compare the simulations under hydrostatic stress with experimental data. In a previous paper (Stangarone et al., 2019) we have shown that the simulations under hydrostatic stress slightly over-estimate the unit-cell volume by $0.6 \%$, and the $\mathrm{Si}-\mathrm{O}$ and the shorter $\mathrm{Zr}-\mathrm{O}$ bond distances by $0.7-0.8 \%$. This arises from the small error in calculating electron self-interactions in the core regions of the atoms (Cremer, 2001) in the simulations. Nonetheless, the important distortions of the $\mathrm{SiO}_{4}$ tetrahedra are well-reproduced, the predicted structural evolution with pressure is consistent with the limited experimental data (Hazen \& Finger, 1979), and the elastic anisotropy of the structure is correct in the DFT simulations. Similarly, both the Raman shifts calculated at zero pressure (Table 1) and their change with 


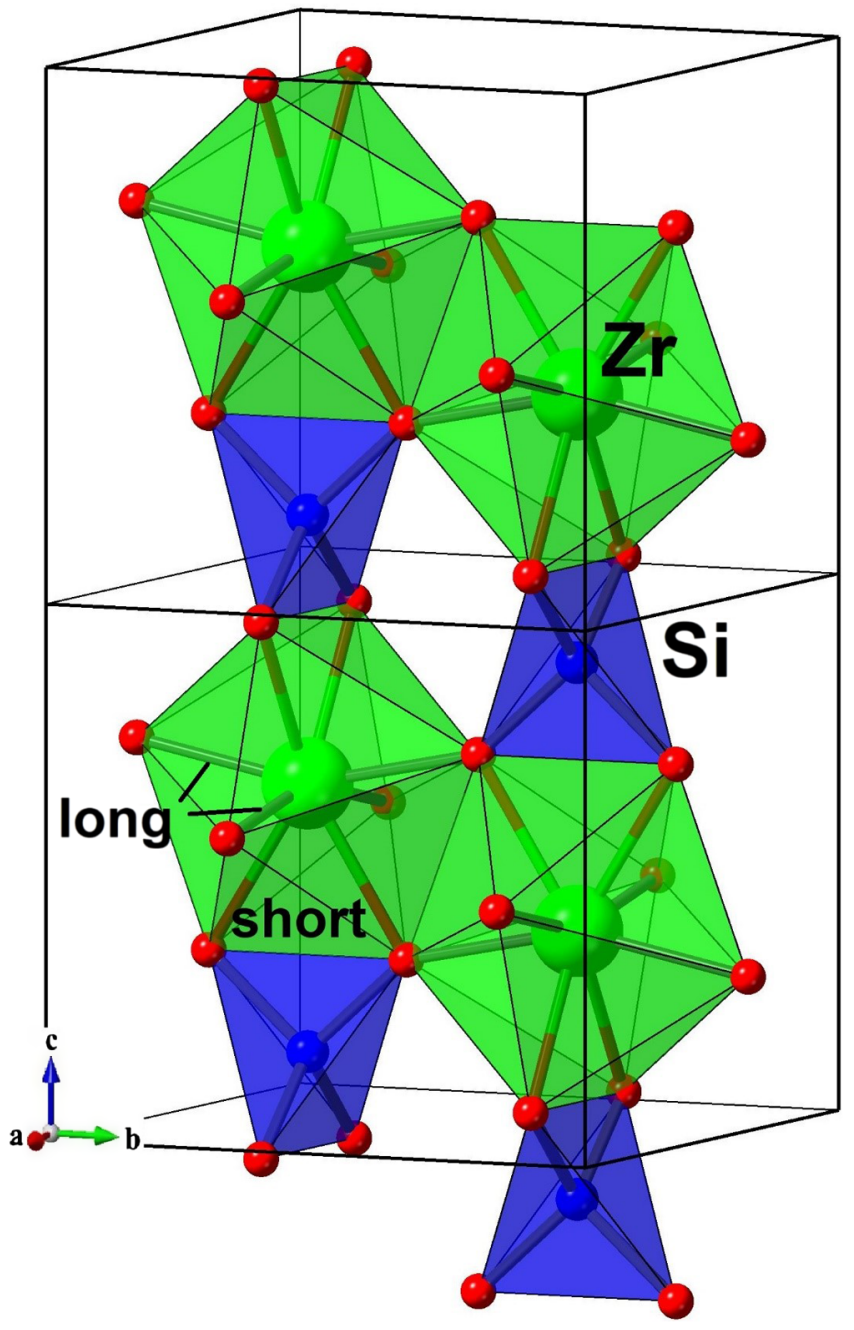

Fig. 1. Polyhedral representation of the crystal structure of zircon, showing two adjacent columns of alternating edge-sharing $\mathrm{ZrO}_{8}$ and $\mathrm{SiO}_{4}$ polyhedra. The long and short $\mathrm{Zr}-\mathrm{O}$ bonds are indicated. Drawn with CrystalMaker ${ }^{\circledR}$ (2018).

pressure are in good agreement with experimental data (Syme et al., 1977; Pina-Binvignat et al., 2018).

Figure 2 is a series of contour plots of structural parameters of zircon to show how they respond to the strains $\varepsilon_{1}$ and $\varepsilon_{3}$. Under non-hydrostatic conditions the length of the $\mathrm{O}-\mathrm{O}$ shared edge between the $\mathrm{SiO}_{4}$ tetrahedra and the $\mathrm{ZrO}_{8}$ dodecahedra, which is perpendicular to the c-axis (Fig. 1), is almost unchanged under compression of the c-axis, but it responds to strains $\varepsilon_{1}$ in the $\mathbf{a}-\mathbf{b}$ plane (Fig. 2a). Thus, when the $\mathbf{c}$-axis is compressed $\left(\varepsilon_{3}<0\right)$ the distance between adjacent $\mathrm{Si}$ and $\mathrm{Zr}$ is reduced by the same amount as the unit-cell strain, the $\mathrm{O}-\mathrm{O}$ edge moves towards the $\mathrm{Si}$, and thus the corresponding $\mathrm{O}-\mathrm{Si}-\mathrm{O}$ angle opens and increases towards the ideal tetrahedral angle of $109.47^{\circ}$. As a consequence, the distortion of the $\mathrm{SiO}_{4}$ tetrahedra as measured by their angle variance (Robinson et al., 1971) decreases significantly with compression along the c-axis, but is not changed by strains $\varepsilon_{1}$ (Fig. 2b). The length of the $\mathrm{Zr}-\mathrm{O}$ long bonds to the $\mathrm{O}$ atoms in the shared edge is also therefore
Table 1. Calculated and observed phonon-modes for zircon.

\begin{tabular}{|c|c|c|c|c|c|}
\hline Symmetry & $\begin{array}{l}\omega_{0, \mathrm{DFT}}^{m} \\
\mathrm{~cm}^{-1}\end{array}$ & $\begin{array}{l}\text { Raman } \\
\text { active }\end{array}$ & $\begin{array}{c}\text { IR } \\
\text { active }\end{array}$ & $\begin{array}{l}\omega_{0,0 \mathrm{~K}}^{m} \\
\mathrm{~cm}^{-1}\end{array}$ & $\begin{array}{c}\omega_{0,90 \mathrm{~K}}^{m} \\
\mathrm{~cm}^{-1}\end{array}$ \\
\hline & & $\mathrm{A}=$ active & $\mathrm{A}=$ active & Note 1 & Note 2 \\
\hline$B_{1 \mathrm{u}}$ & 130.6 & & & & \\
\hline$E_{\mathrm{g}}$ & 196.9 & A & & $201.0(2)$ & $203(2)$ \\
\hline$B_{1 \mathrm{~g}}$ & 213.6 & A & & $215.0(1)$ & $217(2)$ \\
\hline$E_{\mathrm{g}}$ & 223.1 & A & & $224.2(2)$ & $225(2)$ \\
\hline$A_{2 \mathrm{~g}}$ & 238.8 & & & & \\
\hline$B_{2 \mathrm{~g}}$ & 247.8 & A & & & $265(2)$ \\
\hline$E_{\mathrm{u}}$ & 268.2 & & A & & \\
\hline$A_{2 \mathrm{u}}$ & 332.1 & & A & & \\
\hline$E_{\mathrm{g}}$ & 341.5 & A & & $358.8(2)$ & $360(2)$ \\
\hline$E_{\mathrm{u}}^{\mathrm{g}}$ & 370.8 & & A & & \\
\hline$B_{1 \mathrm{~g}}$ & 386.5 & A & & $394.5(2)$ & $395(4)$ \\
\hline$A_{1 \mathrm{u}}$ & 397.1 & & & & \\
\hline$E_{\mathrm{u}}$ & 420.4 & & A & & \\
\hline$A_{1 \mathrm{~g}}$ & 437.7 & A & & $441.2(2)$ & $442(4)$ \\
\hline$E_{\mathrm{g}}$ & 541.0 & A & & $546.8(4)$ & $547(4)$ \\
\hline$B_{2 \mathrm{u}}$ & 566.6 & & & & \\
\hline$A_{2 \mathrm{u}}$ & 599.5 & & A & & \\
\hline$B_{1 \mathrm{~g}}$ & 635.6 & A & & $640.5(6)$ & $642(3)$ \\
\hline$E_{\mathrm{u}}$ & 862.7 & & A & & \\
\hline$E_{\mathrm{g}}$ & 921.0 & A & & $927(2)$ & $927(4)$ \\
\hline$B_{2 \mathrm{u}}$ & 938.4 & & & & \\
\hline$A_{1 \mathrm{~g}}$ & 969.0 & A & & $977.1(2)$ & $978(3)$ \\
\hline$A_{2 \mathrm{u}}$ & 980.5 & & A & & \\
\hline$B_{1 \mathrm{~g}}$ & 1014.4 & A & & $1012.6(4)$ & 1013(3) \\
\hline
\end{tabular}

\section{Notes:}

(1) Estimated from a 4th-order polynomial fit of experimentally measured wavenumbers versus $T$ measured over the range from 80 to $1400 \mathrm{~K}$ (see Supplementary Material).

(2) Data from Syme et al. (1977).

dependent solely on the strain $\varepsilon_{3}$ (Fig. 2c). In contrast, because the shorter $\mathrm{Zr}-\mathrm{O}$ bonds to polyhedral edges shared between adjacent $\mathrm{ZrO}_{8}$ polyhedra (Fig. 1) are sub-parallel to (001), their length is dependent mostly on the strain $\varepsilon_{1}$ and is insensitive to $\varepsilon_{3}$ (Fig. 2d). It is the combination of these two different responses of the long and short $\mathrm{Zr}-\mathrm{O}$ bonds to applied strains, induced by the $\mathrm{Zr}-\mathrm{Si}$ repulsion and the stiffness of the $\mathrm{O}-\mathrm{O}$ shared edge, that leads to the volumes of both the $\mathrm{SiO}_{4}$ tetrahedra and the $\mathrm{ZrO}_{8}$ polyhedra (Fig. 2e, f) following the isochors of the unit-cell volume (lines of constant $2 \varepsilon_{1}+\varepsilon_{3}$ ) while their distortions (Fig. $2 \mathrm{~g}$ ) are almost entirely dependent on the deviatoric strain $\left(\varepsilon_{3}-\varepsilon_{1}\right)$. In summary, the anisotropy of response of this $\mathrm{O}-\mathrm{O}$ shared edge, which must be due to the $\mathrm{Si}-\mathrm{Zr}$ repulsion, is responsible for the anisotropy of response of the other structural parameters.

\subsection{Vibrational modes}

The wavenumbers of all of the phonon modes in zircon calculated for the structure at the static equilibrium (zero $T$ and $P$ and with no zero-point pressure included) are listed in Table 1 as $\omega_{0, \mathrm{DFT}}^{m}$. As previously reported in Stangarone et al. (2019), they are in reasonable agreement with the values estimated by extrapolating to $0 \mathrm{~K}$ the experimental 


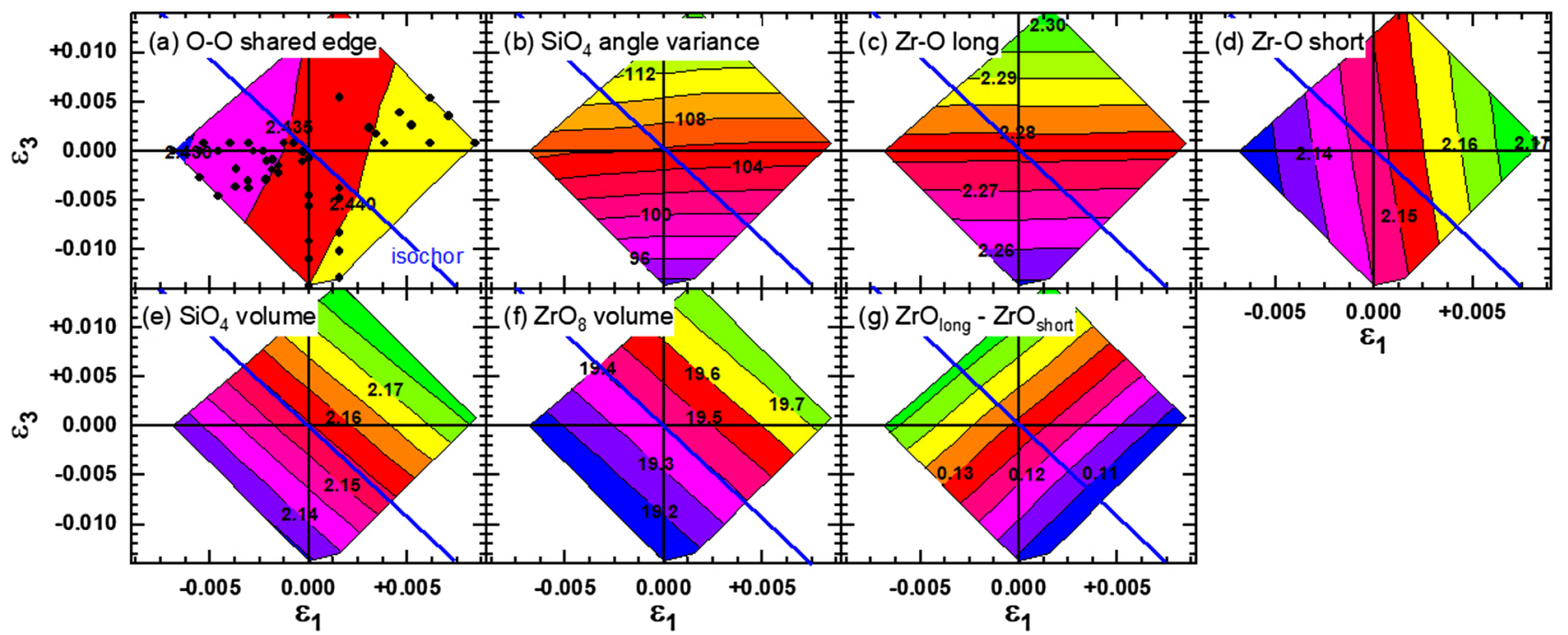

Fig. 2. Variation of structural parameters of zircon with non-symmetry-breaking strains $\varepsilon_{1}$ and $\varepsilon_{3}$. Expansion is towards the top right, compression towards the bottom left in each plot. The top left and bottom right quadrants represent conditions of shear. All contours are equally spaced and are interpolated on the underlying data. Contours of distances are labelled in $\AA$, angle variance in degrees, and volumes in $\AA^{3}$. The blue line in each plot is an isochor. The black dots in part (a) indicate the strain conditions at which DFT structure optimisations were performed.

values measured over the range of 80-1400 K (full details in the Supplementary Material of the present paper). The level of discrepancy is similar to that obtained in previous simulations of the Raman spectra of zircon (Sheremetyeva et al., 2018) and other silicate minerals (Prencipe, 2019). For ease of understanding we will refer to the modes by their wavenumbers as measured experimentally at room conditions, and their symmetries (Table 2).

Figure 3 shows contour plots of the calculated changes in the wavenumbers of five phonon modes that have been selected to illustrate the range of behaviour found in zircon. The contours are lines of equal change in the wavenumbers of the phonon modes from the unstrained reference state, and in Fig. 3 they are simply interpolated between the calculated wavenumbers of the DFT simulations, without fitting a model. They join strain states that have the same wavenumber for the phonon mode and thus, if they are Raman-active modes, these strained crystals will exhibit the same Raman shift. As a consequence, it is not possible to determine the strain, or the stress, in an inclusion by measuring the position of a single Raman peak. We call the contours "isoshift lines". All of the phonon modes exhibit isoshift lines that are approximately linear, parallel and equally spaced (Fig. 3). Different modes have different slopes of the isoshift lines with respect to the strain axes; for example the $E_{\mathrm{g}}$ mode near $356 \mathrm{~cm}^{-1}$ clearly responds more to $\varepsilon_{1}$ than $\varepsilon_{3}$, whereas the opposite is true for the $E_{\mathrm{g}}$ mode near $224 \mathrm{~cm}^{-1}$ (Fig. 3b, c). In general, the isoshift lines are not parallel to isochors, which are lines of constant $2 \varepsilon_{1}+\varepsilon_{3}$ (e.g., Fig. 3b). The variation of slopes of the isoshift lines between modes also clearly means that they are not, in general, parallel to isobars which are lines of equal average stress $\left(2 \sigma_{1}+\sigma_{3}\right) / 3$. This is a general behaviour for crystals, which we have previously described for quartz (Murri et al.,
2019). It indicates that the phonon-mode wavenumbers change approximately linearly with applied strains and can therefore be described by the phonon-mode Grüneisen tensor $\gamma^{m}$ (e.g., Ziman, 1960; Barron et al., 1980; Angel et al., 2019) which is a linear model. For uniaxial crystals in which the symmetry is not broken by the strain (as we have for our DFT simulations), $\varepsilon_{1}=\varepsilon_{2}$ and the wavenumber change $\Delta \omega^{m}$ is given by:

$\frac{-\Delta \omega^{m}}{\omega_{0}^{m}}=2 \gamma_{1}^{m} \varepsilon_{1}+\gamma_{3}^{m} \varepsilon_{3}$,

in which $\omega_{0}^{m}$ is the wavenumber of the phonon mode in the unstrained reference state, and $\gamma_{1}^{m}$ and $\gamma_{2}^{m}=\gamma_{3}^{m}$ are the only two non-zero independent components of the phononmode Grüneisen tensor (Angel et al., 2019). The values of $\gamma_{1}^{m}$ and $\gamma_{3}^{m}$ (Table 2, and cif in Supplementary Material) were determined for each mode by a least-squares fit of Equation (1) to the values of $\Delta \omega^{m}$ determined from the 47 DFT simulations at different strain states, using the unstrained simulation as the reference state for the calculation of both the strains and $\Delta \omega^{m}$. Equation (1) shows that the values of $\gamma_{1}^{m}$ and $\gamma_{3}^{m}$ calculated in this way depend on both the observed $\Delta \omega^{m}$ and the $\omega_{0}^{m}$ of the reference state (Angel et al., 2019; Murri et al., 2019). Because $\gamma_{1}^{m}$ and $\gamma_{3}^{m}$ will be used to calculate changes in phonon-mode wavenumbers in zircon relative to room conditions, and not to $0 \mathrm{~K}$, their values (Table 2) have been calculated not with $\omega_{0, \mathrm{DFT}}^{m}$ but with the experimentally observed wavenumber at room conditions, $\omega_{0,298 \mathrm{~K}}^{m}$.

Consideration of Table 2 together with the isoshift lines of selected modes in Fig. 3 illustrates some general points about phonon-mode Grüneisen tensors. The different slopes of the isoshift lines for different modes in Fig. 3 correspond 
Table 2. Characteristics of selected phonon modes of zircon.

\begin{tabular}{|c|c|c|c|c|c|c|c|c|}
\hline$\omega_{0, \mathrm{DFT}}^{m}\left(\mathrm{~cm}^{-1}\right)$ & $\begin{array}{c}\omega_{0,298 \mathrm{~K}}^{m}\left(\mathrm{~cm}^{-1}\right) \\
\text { Note } 1\end{array}$ & Symmetry & \multicolumn{3}{|c|}{$\begin{array}{l}\% \text { of motion } \\
\text { (according to } \\
\text { cation mass } \\
\text { substitution) }\end{array}$} & Atomic motions & $\begin{array}{c}\gamma_{1}^{\mathrm{m}} \\
\text { Note } 2\end{array}$ & $\gamma_{3}^{\mathrm{m}}$ \\
\hline 130.6 (Note 3$)$ & Inactive & $\mathrm{B}_{1 \mathrm{u}}$ & 0 & 0 & 100 & $\begin{array}{l}\text { Rigid rotation of the } \mathrm{SiO}_{4} \text {. No motion } \\
\text { of } \mathrm{Zr} \text { or Si}\end{array}$ & $-6.5(1)$ & $-6.1(2)$ \\
\hline 213.6 & 213.4 & $B_{1 \mathrm{~g}}$ & 96 & 3 & 1 & Gliding of the $\mathrm{Zr}$ along the c-axis & $1.22(2)$ & $3.44(3)$ \\
\hline 223.1 & 224.0 & $E_{\mathrm{g}}$ & 56 & 7 & 37 & $\begin{array}{l}\text { Rigid rotation of the } \mathrm{SiO}_{4} \text { around the } \\
\text { a-axis, shearing of the } \mathrm{Zr} \text { in }(001)\end{array}$ & $-0.27(2)$ & $1.31(3)$ \\
\hline 247.8 & 265.5 & $B_{2 g}$ & 0 & 0 & 100 & $\begin{array}{l}\text { Anti-symmetric twist of the } \mathrm{SiO}_{4} \\
\text { and } \mathrm{ZrO}_{8} \text { around the c-axis. }\end{array}$ & $-1.19(2)$ & $-0.43(2)$ \\
\hline 341.5 & 356.0 & $E_{\mathrm{g}}$ & 27 & 19 & 54 & $\begin{array}{l}\text { Rigid rotation of the } \mathrm{SiO}_{4} \text { around the } \\
\text { a-axis, shearing of the } \mathrm{Zr} \text { in }(001)\end{array}$ & $3.785(10)$ & $1.337(13)$ \\
\hline 541.0 & 545.5 & $E_{\mathrm{g}}$ & 4 & 28 & 68 & $\begin{array}{l}\text { Anti-symmetric stretching of } \mathrm{SiO}_{4} \text { groups } \\
\text { with } \mathrm{Si} \text { translation }\end{array}$ & $0.121(4)$ & $0.939(6)$ \\
\hline 635.6 & 641.2 & $B_{1 \mathrm{~g}}$ & 1 & 34 & 65 & $\begin{array}{l}\text { Anti-symmetric bending of opposite } \\
\mathrm{O}_{\text {sh }}-\mathrm{Si}-\mathrm{O}_{\text {sh }} \text { angles in each } \mathrm{SiO}_{4} \text {, generating } \\
\text { anti-symmetric } \mathrm{Zr}-\mathrm{O} \text { stretching in } \mathrm{ZrO}_{8}\end{array}$ & $0.852(5)$ & $0.243(7)$ \\
\hline 921.0 & 923.2 & $E_{\mathrm{g}}$ & 0 & 37 & 63 & $\begin{array}{l}\text { Anti-symmetric stretching between } \mathrm{Si} \text { and the } \\
\text { edge sharing oxygen atoms with the } \mathrm{Zr}-\mathrm{O} \\
\text { polyhedra }\end{array}$ & $1.035(3)$ & $2.122(4)$ \\
\hline 969.0 & 974.8 & $A_{1 \mathrm{~g}}$ & 0 & 0 & 100 & $\begin{array}{l}\text { Symmetric stretching of } \mathrm{Si}-\mathrm{O} \text {, generating } \\
\text { stretching of } \mathrm{Zr}-\mathrm{O} \text { and bending of } \mathrm{O}-\mathrm{Zr}-\mathrm{O} \text { in } \\
\mathrm{ZrO}_{8}\end{array}$ & $1.161(3)$ & $1.256(4)$ \\
\hline
\end{tabular}

\section{Notes:}

(1) Determined by fitting experimental Raman data measured over the temperature range from 80 to $1400 \mathrm{~K}$ (see Supplementary Material). Values agree within experimental uncertainties with other published experimental data (Syme et al., 1977; Kolesov et al., 2001; Schmidt et al., 2013), see also Fig. 5.

(2) The values of the phonon-mode Grüneisen tensor components given in this table were determined in combination with the values of $\omega_{0,298 \mathrm{~K}}^{m}$ given in the second column. Therefore, this value of $\omega_{0,298 \mathrm{~K}}^{m}$ must be used in combination with the $\gamma_{i}^{m}$ in order to correctly calculate strains from experimental wavenumber shifts $\Delta \omega_{\exp }^{m}=\omega_{\exp }^{m}-\omega_{0, \text { exp }}^{m}$.

(3) The $B_{1 \mathrm{u}}$ mode at $\omega_{0, \mathrm{DFT}}^{m}=130.6 \mathrm{~cm}^{-1}$ is neither Raman nor infra-red active, so $\omega_{0,298 \mathrm{~K}}^{131}$ cannot be measured. Therefore the values of $\gamma_{1}^{131}$ and $\gamma_{3}^{131}$ have been calculated using $\omega_{0, \mathrm{DFT}}^{m}$.

to significantly different values for $\gamma_{1}^{m}$ and $\gamma_{3}^{m}$. The majority of modes have positive values for both $\gamma_{1}^{m}$ and $\gamma_{3}^{m}$ which means (Eq. (1)) that the phonon-mode wavenumbers increase with negative strains and decrease with positive strains (Fig. 3a, b). This corresponds to the normal behaviour observed in minerals, that the wavenumbers of phonon modes increase under pressure and decrease with increasing temperature. There is also a general trend for the values of $\gamma_{1}^{m}$ and $\gamma_{3}^{m}$ of the modes in zircon to become more similar in magnitude with increasing wavenumber of the mode (Table 2). This decrease in anisotropy of the mode Grüneisen tensors is due to the decreasing contribution of $\mathrm{Zr}$ atom motions to the higher frequency modes which become dominated by the "internal" modes of the $\mathrm{SiO}_{4}$ tetrahedra.
In contrast, the $B_{1 \mathrm{u}}$ mode near $131 \mathrm{~cm}^{-1}$ (which is neither Raman nor infra-red active) corresponds to a rigid rotation of the $\mathrm{SiO}_{4}$ tetrahedra around the c-axis, and has very large negative values $\sim-6$ for $\gamma_{1}^{131}$ and $\gamma_{3}^{131}$ (Fig. 3e) because it is the soft mode that drives the displacive phase transition from zircon to a high-pressure phase with lower symmetry (Stangarone et al., 2019). The large negative values indicate that the wavenumber of this mode decreases strongly with increasing pressure (Fig. 4). The $B_{2 \mathrm{~g}}$ mode near $265 \mathrm{~cm}^{-1}$ and the $E_{\mathrm{g}}$ mode near $201 \mathrm{~cm}^{-1}$ also have both phononmode Grüneisen tensor components negative, but they are small in magnitude (Table 2). This indicates that they are modes that soften because they either couple with the soft mode directly or with the strain. Direct coupling may be 


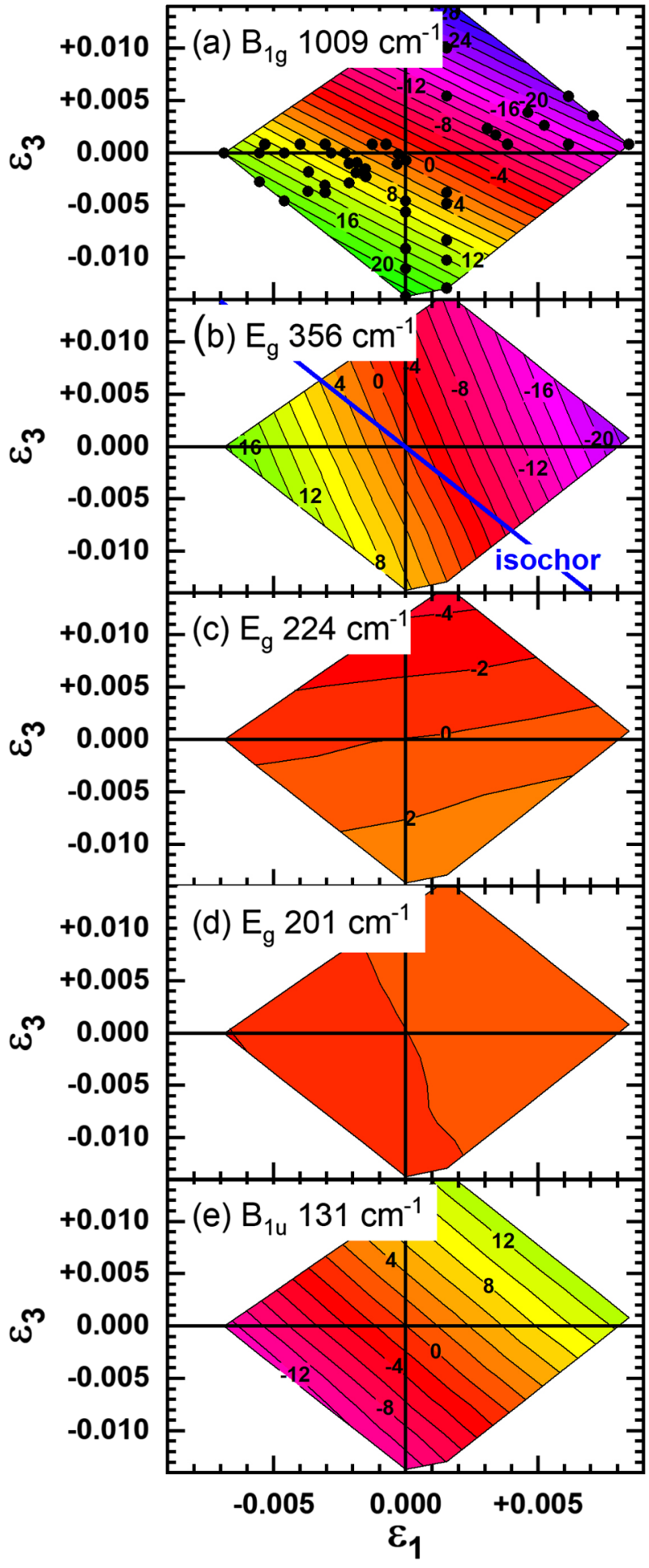

Fig. 3. Contour maps of the calculated change of the wavenumbers of selected phonon modes of zircon with non-symmetry-breaking strains $\varepsilon_{1}$ and $\varepsilon_{3}$. Contours were interpolated on the calculated mode wavenumbers without any model. They are drawn with the same interval of $2 \mathrm{~cm}^{-1}$ in each map, and are labelled in $\mathrm{cm}^{-1}$. The same colour scale is used for all plots. The black dots in part (a) indicate the strain conditions at which DFT simulations were performed. The blue line in part (b) is the isochor of the volume of the reference structure. It is clear that the wavenumber of this mode changes significantly along an isochor.

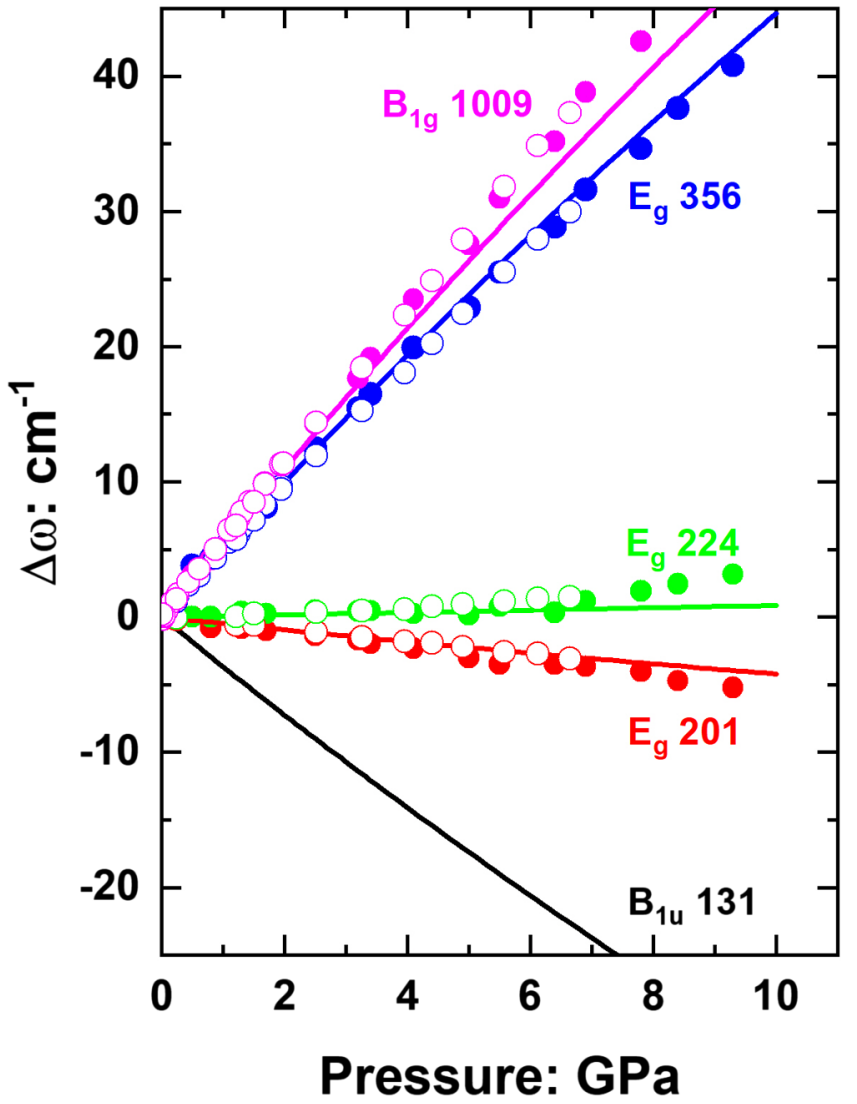

Fig. 4. Comparison of the pressure variation of selected phonon modes of zircon at room temperature predicted from the phononmode Grüneisen tensors (Table 2) and the variation of cell parameters with pressure (Zaffiro, 2019) shown as lines, with experimental data (open symbols, Schmidt et al., 2013; filled symbols, Pina-Binvignat et al., 2018). The $B_{1 \mathrm{u}}$ mode at $131 \mathrm{~cm}^{-1}$ is Raman inactive and cannot be measured with conventional Raman spectroscopy. The other modes not included in the figure show similar agreement between prediction and experiment.

expected in this case, because both of these modes also involve rotations (or twists) of the $\mathrm{SiO}_{4}$ tetrahedra around the $\mathbf{c}$-axis as does the $\mathrm{B}_{1 \mathrm{u}}$ soft mode. Similarly, the $E_{\mathrm{g}}$ mode at $224 \mathrm{~cm}^{-1}$ also involves rigid rotations of the $\mathrm{SiO}_{4}$ tetrahedra (Table 2) and has $\gamma_{1}^{224}<0$ and $\gamma_{3}^{224}>0$. The phononmode Grüneisen tensors therefore clearly distinguish the so-called "hard modes" (e.g., Salje, 1992; Salje \& Bismayer, 1997) which have relatively small positive components $\gamma_{i}^{m}$ from soft modes that drive displacive phase transitions to low-symmetry phases at high pressure (large negative $\gamma_{i}^{m}$ ), and the modes that are affected by, or support, the same phase transitions (small negative $\gamma_{i}^{m}$ ).

This analysis is based (Eq. (1)) on a linear relationship between the changes in wavenumbers of the phonon modes and the strains, while the DFT simulations show (Fig. 3) that there are small non-linearities in the variation of wavenumbers with strain. It therefore should be understood that the values of $\gamma_{1}^{m}$ and $\gamma_{3}^{m}$ determined from the DFT simulations are average values over the range of strains included in the analysis. Whether the values are correct, and whether 


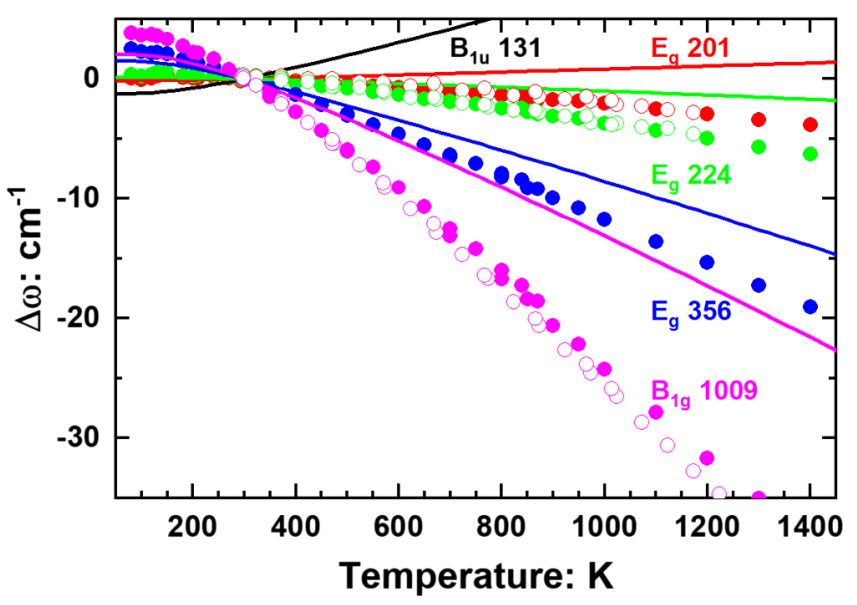

Fig. 5. Comparison of the temperature variation of selected phonon modes of zircon at room pressure calculated from the phonon-mode Grüneisen tensors (Table 2) and the variation of cell parameters with temperature (Zaffiro, 2019) shown as lines, with experimental data (open symbols, Schmidt et al. (2013); filled symbols, this work, data in Supplementary Material). The $B_{1 \mathrm{u}}$ mode at $131 \mathrm{~cm}^{-1}$ is Raman inactive and cannot be measured with conventional Raman spectroscopy. The other modes not included in the figure show similar differences between prediction and experiment.

they can be used for larger strains, can only be determined by comparison with experimental data. We can use the known EoS and cell-parameter variation of zircon (Zaffiro, 2019) to calculate the strains relative to room conditions induced by $P$ and/or $T$, and from the calculated strains predict the change in the phonon wavenumber from Equation (1). Figure 4 shows that the Grüneisen tensor approach correctly predicts the shifts of the phonon modes with pressure, including the $E_{\mathrm{g}}$ modes at $201 \mathrm{~cm}^{-1}$ and $224 \mathrm{~cm}^{-1}$ that are coupled to the soft $B_{1 \mathrm{u}}$ mode. A limit to the linear relationship might be indicated by the small discrepancies between predicted and observed phonon-mode wavenumber changes when $\Delta \omega^{m}>20 \mathrm{~cm}^{-1}$ or $P>7 \mathrm{GPa}$, whichever occurs first.

However, Fig. 5 shows that the experimental measurements of the phonon-mode wavenumbers at both low and high temperatures at room pressure are not predicted by the mode Grüneisen tensor components determined from the DFT simulations. For the hard modes the decrease in wavenumber with increasing temperature is underestimated, by different amounts for the various modes. For the phonon modes with negative $\gamma_{i}^{m}$ the positive strains associated with thermal expansion mean that the Grüneisen tensor approach predicts that these modes should exhibit a positive wavenumber change with increasing temperature, whereas the opposite is observed experimentally for the $E_{\mathrm{g}}$ mode at $201 \mathrm{~cm}^{-1}$.

\section{Discussion}

Phonon-mode Grüneisen tensor coefficients calculated from the results of DFT simulations predict the experimentally measured mode-wavenumber changes of zircon with $P$ at room $T$ (Fig. 4), which shows that the results of the DFT simulations are accurate. Therefore, the failure to correctly predict the mode shifts induced by $T$ alone (Fig. 5) must arise from the application of the concept of the phononmode Grüneisen tensor. This result is very different from quartz, where the phonon-mode Grüneisen tensor components correctly predict the changes in the wavenumbers of Raman modes with both $P$ and $T$, at least at conditions where the effects of the alpha-beta phase transition do not dominate the behaviour of quartz (Murri et al., 2018, 2019).

This contrast in the behaviour of the Raman modes of quartz and zircon can be understood as the consequence of differences in the anisotropies of their compressibility and thermal expansion.

When the strains in a crystal are induced by a change in hydrostatic pressure at constant temperature, we can rewrite Equation (1) in terms of the axial compressibilities $\beta_{i}=\frac{-1}{l_{i}} \cdot \frac{\mathrm{d} l_{i}}{\mathrm{~d} P}$

$$
\left(\frac{\partial \omega^{m}}{\partial P}\right)_{T}=\left(2 \gamma_{1}^{m} \beta_{1}+\gamma_{3}^{m} \beta_{3}\right) \omega_{0}^{m}
$$

Hazen \& Finger (1982) established that, in general, directions in minerals that are stiff and therefore have large linear moduli and small values of linear compressibility, tend also to be the directions of relatively low linear thermal expansion. This makes intuitive sense in a simplistic way; stiffer directions in a mineral structure are often associated with chains of strong inter-atomic bonds, and the strong bonding might be expected to lead to low values of linear thermal expansion. ${ }^{1}$ If the ratio of the linear thermal expansion coefficient $\alpha_{i}=\frac{1}{l_{i}} \cdot \frac{\mathrm{d} l_{i}}{\mathrm{~d} T}$ to the compressibility is the same for all directions $i$ in a crystal, then in uniaxial crystals such as zircon and quartz:

$\frac{\alpha_{1}}{\beta_{1}}=\frac{\alpha_{3}}{\beta_{3}}=\frac{\alpha_{V}}{\beta_{V}}$.

Within $1 \%$ this is true for quartz at room conditions (Table 3). Substitution from (3) into (2) gives:

$$
\left(\frac{\partial \omega^{m}}{\partial P}\right)_{T}=\left(2 \gamma_{1}^{m} \alpha_{1}+\gamma_{3}^{m} \alpha_{3}\right)\left(\frac{\beta_{V}}{\alpha_{V}}\right) \omega_{0}^{m}
$$

Comparison to the expression for the effect of isobaric temperature change on the phonon wavenumbers (Angel et al., 2019) which also follows from Equation (1):

$$
\left(\frac{\partial \omega^{m}}{\partial T}\right)_{P}=-\left(2 \gamma_{1}^{m} \alpha_{1}+\gamma_{3}^{m} \alpha_{3}\right) \omega_{0}^{m}
$$

shows that, in this particular case of isotropy, the relationship between the isobaric and isothermal changes in phonon wavenumbers is:

\footnotetext{
1 Of course this is an overly simplistic view. Stiffness in a given direction in a crystal structure is due to the mutual repulsion of adjacent atoms, not to their attraction, and it is a property of the static structure. Whereas the thermal expansivity is related to the anharmonicity of the inter-atomic interactions and the strength of the bonding and is therefore an intrinsically dynamic property!
} 
Table 3. Thermo-elastic properties of zircon and quartz at room conditions.

\begin{tabular}{lllc}
\hline & a-axis & c-axis & Volume \\
\hline & & Zircon & \\
\hline $\mathrm{M}_{T}, \mathrm{~K}_{T}: \mathrm{GPa}$ & 578 & 1009 & 225 \\
$\beta: \mathrm{TPa}^{-1}$ & 1.73 & 0.99 & 4.45 \\
$\alpha: 10^{5} \mathrm{~K}^{-1}$ & 0.29 & 0.49 & 1.07 \\
$\alpha / \beta: \square \mathrm{Pa} \mathrm{K}$ & 1.71 & 4.99 & 2.400 \\
& & Quartz & \\
$M_{T}, K_{T}: \mathrm{GPa}$ & 102 & 137 & 37.1 \\
$\beta: \mathrm{TPa}^{-1}$ & 9.80 & 7.31 & 26.9 \\
$\alpha: 10^{5} \mathrm{~K}^{-1}$ & 1.36 & 1.02 & 3.74 \\
$\alpha / \beta: \mathrm{MPa} \mathrm{K}^{-1}$ & 1.38 & 1.40 & 1.39 \\
\hline
\end{tabular}

Notes: Volume EoS and anisotropic properties of zircon from Zaffiro (2019). Volume EoS of quartz from Angel et al. (2017a) and anisotropic properties from Alvaro et al., submitted.

$$
\left(\frac{\partial \omega^{m}}{\partial P}\right)_{T}=-\left(\frac{\beta_{V}}{\alpha_{V}}\right)\left(\frac{\partial \omega^{m}}{\partial T}\right)_{P}
$$

Because we have eliminated the phonon-mode Grüneisen tensor components $\gamma_{1}^{m}$ and $\gamma_{3}^{m}$ from the equations without making any further assumptions, we have shown that when a crystal exhibits isotropy in $\alpha_{i} / \beta_{i}$ (Eq. (3)), the same phonon-mode Grüneisen tensor can explain the changes in phonon-mode wavenumbers induced by changes in either $P$ or $T$, as found for many modes in quartz (Murri et al., 2018). Similarly, it will always apply to cubic materials, for which Equation (3) is always true.

However, the c-axis of zircon is considerably stiffer than the a-axis and also has a higher thermal expansion coefficient (Table 3), as a consequence of the $\mathrm{Zr}-\mathrm{Si}$ repulsion across the shared $\mathrm{O}-\mathrm{O}$ polyhedral edge. Therefore Equation (3) does not hold for zircon and therefore Equation (4) and the subsequent equations cannot be derived, which means that the thermally induced changes in phonon-mode wavenumbers in zircon are independent and different from the wavenumber changes induced by hydrostatic pressure. However, the changes in phonon-mode wavenumbers induced by pressure (Fig. 4), or strain, at any fixed temperature are still correctly predicted by the phonon-mode Grüneisen tensor, meaning that we can still use the concept to determine isothermal strains in crystals from the measured Raman shifts.

\section{Conclusions}

Our determination of the phonon modes of zircon under various strains which deviate from hydrostatic stress conditions has shown that, if the temperature is held constant, the changes in their wave numbers depends approximately linearly on the unit-cell strains, up to strains of a few \% (Figs. 3, 4). This linear dependence is described by the components of the phonon-mode Grüneisen tensors of zircon, which we have reported for all of the Raman-active bands (Table 2). Because of the strong anisotropy of the ratio of linear thermal expansion to linear compressibility (the anisotropic thermal pressure tensor; Nye, 1957) in zircon, these phonon-mode Grüneisen tensors do not describe the temperature-induced shifts in the phonon-mode wavenumbers (Fig. 5). Nonetheless, they do correctly predict the changes in phonon wavenumbers with isothermal changes in hydrostatic pressure (Fig. 4), and should therefore also apply to conditions of non-hydrostatic stress. Thus, the values of $\gamma_{1}^{m}$ and $\gamma_{3}^{m}$ that we have determined for zircon (Table 2) can be used to map the residual strains in zircon inclusions trapped inside garnets, and other host minerals that are almost elastically isotropic.

For example, Fig. 6 shows the strains calculated from Raman spectra collected on two traverses across a zircon inclusion trapped about $1 \mathrm{~cm}$ from the rim of a $12 \mathrm{~cm}-$ diameter pyrope megablast from the whiteschists of the UHP Brossasco-Isasca Unit in the Dora-Maira massif originally described by Chopin (1984). The full Raman spectrum at each point was measured (Fig. 6b). The Raman shift of the $356 \mathrm{~cm}^{-1} E_{\mathrm{g}}$ mode along the two traverses of the inclusion (Fig. 6c) is clearly different and this shows immediately that the strain in the inclusion is inhomogeneous. A total of seven Raman peaks from zircon are clearly resolved from the spectra of the garnet host (Fig. 6b). Of these, the three peaks at $200-225 \mathrm{~cm}^{-1}$ and the peak near $439 \mathrm{~cm}^{-1}$ exhibit small shifts with strains as the combination of relatively small coefficients of their phonon-mode Grüneisen tensors (Table 2) and small values of $\omega_{0}^{m}$. This combination of factors means that their measured shifts from the reference crystal do not significantly constrain the values of strains. On the other hand, the Raman-active modes near 356, 975 and $1009 \mathrm{~cm}^{-1}$ are stronger (Fig. 6b) and exhibit larger shifts. The difference in the ratios $\gamma_{1}^{m} / \gamma_{3}^{m}$ for these three modes means that their isoshift lines have different slopes when plotted against the strains $\varepsilon_{1}$ and $\varepsilon_{3}$ (e.g., Fig. 3a, b). Therefore, the strains at any one point in the inclusion can be determined from the changes in wavenumber of these three Raman modes.

The determination of strains from the Raman spectra of inclusions is entirely dependent on the difference in peak wavenumber from that of an unstrained reference crystal. We therefore measured an unstrained reference crystal in air during the same measurement session and calculated the difference in wavenumbers as $\Delta \omega_{\exp }^{m}=\omega_{\text {exp }}^{m}-\omega_{0, \text { exp }}^{m}$. Strains were then calculated from the values of $\Delta \omega_{\exp }^{m}$ by using Equation (1) in a least-squares procedure implemented in the stRAinMAN program (Angel et al., 2019) which is available at www.rossangel.net. The file zircon_gtensor.cif provided in the Supplementary Material for this paper and available on-line contains the values of $\gamma_{1}^{m}$ and $\gamma_{3}^{m}$ for all of the Raman active modes of zircon and can be used to perform this calculation. In this way, the strains and their gradients can be mapped across trapped inclusions by Raman spectroscopy (Fig. 6d). It is clear that the strains not only vary significantly between the different points, but the gradient in strains along the crystal is different for $\varepsilon_{1}$ and $\varepsilon_{3}$. This confirms that the strains vary inhomogeneously throughout the inclusion as a consequence of its shape (Mazzucchelli et al., 2018) and elastic anisotropy (Mazzucchelli, 2019), 

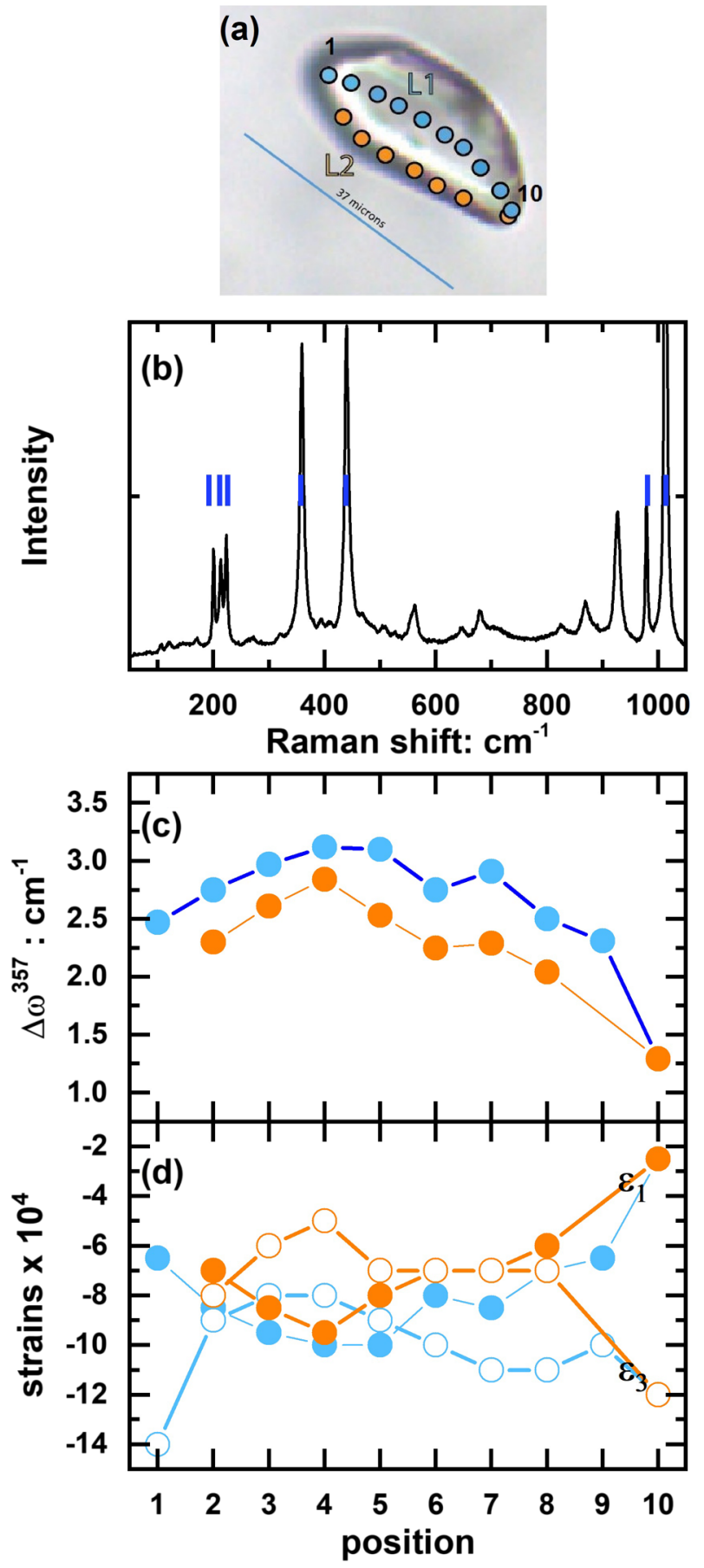

Fig. 6. Raman measurements across a zircon inclusion in a pyrope megablast from the whiteschists of the Dora-Maira UHP BrossascoIsasca Unit. The inclusion is elongated along its crystallographic c-axis. (a) The positions from which the Raman spectra were collected. (b) Example spectrum collected at point 4 on traverse L1, with a Horiba Jobin Yvon Explora_Plus spectrometer, University of Genova, with a quoted spectral resolution of about $2 \mathrm{~cm}^{-1}$. Peaks from zircon are marked by blue vertical lines. (c) The wavenumber shift $\Delta \omega_{\text {exp }}^{m}=\omega_{\text {exp }}^{m}-\omega_{0 \text { exp }}^{m}$ of the $E_{\mathrm{g}}$ mode near $356 \mathrm{~cm}^{-1}$ along the two traverses. Other Raman modes show similar patterns. (d) The strains calculated from the wavenumber shifts of the Raman peaks near 356, 975 and $1009 \mathrm{~cm}^{-1}$. and consequently the inclusion is under inhomogeneous stress, with significant stress gradients.

The interpretation of the strains of the inclusion in terms of entrapment conditions can then be performed two ways. If the inclusion was approximately spherical, or ellipsoidal, then the strains would be anisotropic and homogeneous, and could be converted to stresses. The mean normal stress could then be used as the inclusion pressure within an isotropic calculation to yield the entrapment isomeke (Angel et al., 2014, 2017b) which should approximate the entrapment conditions. A more complete but more complex analysis for inclusions shaped as the one in Fig. 6 requires the anisotropic relaxation of the host-inclusion system to be calculated (Mazzucchelli, 2019) and then the individual strains at the centre of the inclusion, after correction for relaxation, can be combined to calculate the entrapment $P$ and $T$.

Acknowledgements: This work was supported by ERC starting grant 714936 "True Depths" to Matteo Alvaro. MA was also supported by the F.A.R.E.-M.I.U.R. grant R164WEJAHH. We thank Mara Murri and Mattia Mazzucchelli for discussions and Gabriele Zaffiro for the EoS of zircon.

\section{References}

Angel, R.J., Alvaro, M., Miletich, R., Nestola, F. (2017a): A simple and generalised P-T-V EoS for continuous phase transitions, implemented in EosFit and applied to quartz. Contrib. Mineral. Petrol., 172, 29.

Angel, R.J., Mazzucchelli, M.L., Alvaro, M., Nestola, F. (2017b): EosFit-Pinc: a simple GUI for host-inclusion elastic thermobarometry. Am. Mineral., 102, 1957-1960.

Angel, R.J., Mazzucchelli, M.L., Alvaro, M., Nimis, P., Nestola, F. (2014): Geobarometry from host-inclusion systems: the role of elastic relaxation. Am. Mineral., 99, 2146-2149.

Angel, R.J., Murri, M., Mihailova, B., Alvaro, M. (2019): Stress, strain and Raman shifts. Zeit. Kristallogr., 234, 129-140.

Barron, T.H.K., Collins, J.G., White, G.K. (1980): Thermal expansion of solids at low temperatures. Adv. Phys., 29, 609730.

Campomenosi, N., Mazzucchelli, M.L., Mihailova, B., Scambelluri, M., Angel, R.J., Nestola, F., Reali, A., Alvaro, M. (2018): How geometry and anisotropy affect residual strain in host inclusion system: coupling experimental and numerical approaches. Am. Mineral., 103, 2032-2035.

Chopin, C. (1984): Coesite and pure pyrope in high-grade blueschists of the Western Alps: a first record and some consequences. Contrib. Mineral. Petrol., 86, 107-118.

Cremer, D. (2001): Density functional theory: coverage of dynamic and non-dynamic electron correlation effects. Molecular Phys., 99, 1899-1940.

CrystalMaker. (2018): CrystalMaker, a Crystal \& Molecular Structures Modelling Program for Mac \& Windows, www.crystalmaker.com. CrystalMaker Software Ltd, Oxford, U.K.

Dovesi, R., Erba, A., Orlando, R., Zicovich-Wilson, C.M., Civalleri, B., Maschio, L., Rérat, M., Casassa, S., Baima, J., Salustro, S., Kirtman, B. (2018): Quantum-mechanical condensed matter simulations with CRYSTAL. WIREs Comput. Mol. Sci., 8, e1360:1-36. 
Finch, R.J. \& Hanchar, J.M. (2003): Structure and chemistry of zircon and zircon-group minerals. in "Zircon", Hanchar, J.M., Hoskin, P.W.O., eds., Mineralogical Society of America, Washington DC, USA, 1-26.

Gerya, T. (2015): Tectonic overpressure and underpressure in lithospheric tectonics and metamorphism. J. Metamorphic Geol., 33, 785-800.

Gillet, P., Ingrin, J., Chopin, C. (1984): Coesite in subducted continental crust: P-T history deduced from an elastic model. Earth Planet. Sci. Lett., 70, 426-436.

Hazen, R.M. \& Finger, L.W. (1979): Crystal structure and compressibility of zircon at high pressure. Am. Mineral., 64, 196-201.

—, - (1982): Comparative Crystal Chemistry. John Wiley and Sons, New York, 231 p.

Henry, C., Michard, A., Chopin, C. (1993): Geometry and structural evolution of ultra-high-pressure and high-pressure rocks from the Dora-Maira massif, Western Alps Italy. J. Struct. Geol., 15, 965-982.

Kolesov, B.A., Geiger, C.A., Armbruster, T. (2001): The dynamic properties of zircon studied by single-crystal X-ray diffraction and Raman spectroscopy. Eur. J. Mineral., 13, 939-948.

Mazzucchelli, M. (2019): Development of Computational Mechanics Methods for Geobarometry of Non-ideal Host-inclusion Systems. Ph.D. thesis, Department of Earth and Environmental Sciences, Pavia, 214 p.

Mazzucchelli, M.L., Burnley, P., Angel, R.J., Morganti, S., Domeneghetti, M.C., Nestola, F., Alvaro, M. (2018): Elastic geothermobarometry: corrections for the geometry of the hostinclusion system. Geology, 46, 231-234.

Murri, M., Mazzucchelli, M.L., Campomenosi, N., Korsakov, A.V., Prencipe, M., Mihailova, B., Scambelluri, M., Angel, R.J., Alvaro, M. (2018): Raman elastic geobarometery for anisotropic mineral inclusions. Am. Mineral., 103, 1869-1872.

Murri, M., Alvaro, M., Angel, R.J., Prencipe, M., Mihailova, B.D. (2019): The effects of non-hydrostatic stress on the structure and properties of alpha-quartz. Phys. Chem. Minerals, published online. DOI: 10.1007/s00269-018-01018-6.

Nye, J.F. (1957): Physical properties of crystals. Oxford University Press, Oxford, 329p.

Pina-Binvignat, F.A., Malcherek, T., Angel, R.J., Paulmann, C., Schlüter, J., Mihailova, B. (2018): Effect of radiation-induced structural damage on the structural response of zircon to high pressures. Phys. Chem. Minerals, 45, 981-993.

Prencipe, M. (2019): Quantum mechanics in Earth sciences: a onecentury-old story. Rend. Lincei. Sci. Fis. Nat. DOI: 10.1007/ s12210-018-0744-1.

Robinson, K., Gibbs, G., Ribbe, P.H. (1971): Quadratic elongation: a quantitative measure of distortion in coordination polyhedra. Science, 172, 567-570.
Rosenfeld, J.L. \& Chase, A.B. (1961): Pressure and temperature of crystallization from elastic effects around solid inclusion minerals? Am. J. Sci., 259, 519-541.

Salje, E.K.H. (1992): Hard mode spectroscopy: experimental studies of structural phase transitions. Phase Transition, 37, 83-110.

Salje, E.K.H. \& Bismayer, U. (1997): Hard mode spectroscopy: the concept and applications. Phase Transition, 63, 1-75.

Schertl, H.P., Schreyer, W., Chopin, C. (1991): The pyrope-coesite rocks and their country rocks at Parigi, Dora Maira massif, Western Alps - detailed petrography, mineral chemistry and PTpath. Contrib. Mineral. Petrol., 108, 1-21.

Schmalholz, S.M. \& Podladchikov, Y. (2014): Metamorphism under stress: the problem of relating minerals to depth. Geology, $\mathbf{4 2}$, 733-734.

Schmidt, C., Steele-MacInnis, M., Watenphul, A., Wilke, M. (2013): Calibration of zircon as a Raman spectroscopic pressure sensor to high temperatures and application to water-silicate melt systems. Am. Mineral., 98, 643-650.

Sheremetyeva, N., Cherniak, D.J., Watson, E.B., Meunier, V. (2018): Effect of pressure on the Raman-active modes of zircon $\left(\mathrm{ZrSiO}_{4}\right)$ : a first-principles study. Phys. Chem. Minerals, 45, 173-184.

Smith, D. (1984): Coesite in clinopyroxene in the Caledonides and its implications for geodynamics. Nature, 310, 641-644.

Stangarone, C., Angel, R.J., Prencipe, M., Mihailova, B.D., Alvaro, M. (2019): New insights into the zircon-reidite phase transition. Am. Mineral., in press. DOI: 10.1238/am-2019-6827.

Syme, R.W.G., Lockwood, D.J., Kerr, H.J. (1977): Raman spectrum of synthetic zircon $\left(\mathrm{ZrSiO}_{4}\right)$ and thorite $\left(\mathrm{ThSiO}_{4}\right)$. J. Phys. C: Solid State Phys., 10, 1335-1348.

Tremblay, P. (2013): The little mineral that changed everything. Elements, 9, 246-247.

Wu, Z. \& Cohen, R.E. (2006): More accurate generalized gradient approximation for solids. Phys. Rev. B, 73, 235116.

Zaffiro, G. (2019): Elastic Geobarometry: In-situ Single-crystal X-ray Diffraction Measurements of Inclusions Trapped in Host Minerals to Determine the Entrapment Conditions. Ph.D. Thesis, University of Pavia.

Zhang, Y. (1998): Mechanical and phase equilibria in inclusionhost systems. Earth Planet. Sci. Lett., 157, 209-222.

Ziman, J.M. (1960): Electrons and Phonons: The Theory of Transport Phenomena in Solids. Oxford University Press, Oxford, $469 \mathrm{p}$.

Received 8 November 2018

Modified version received 19 February 2019

Accepted 14 March 2019 